\title{
LINEAR SYSTEMS ON EDGE-WEIGHTED GRAPHS
}

\author{
RODNEY JAMES AND RICK MIRANDA
}

\begin{abstract}
Let $R$ be any subring of the reals. We present a generalization of linear systems on graphs where divisors are $R$-valued functions on the set of vertices and graph edges are permitted to have nonnegative weights in $R$. Using this generalization, we provide an independent proof of a Riemann-Roch formula, which implies the Riemann-Roch formula of Baker and Norine.
\end{abstract}

1. Introduction. Let $R$ be any subring of the reals, and let $G$ be a finite connected edge-weighted graph with vertex set $V=\left\{v_{0}, \ldots, v_{n}\right\}$ and nonnegative weight set $W=\left\{w_{i j} \mid i, j=0, \ldots, n\right\}$, where each $w_{i j} \in R$. Multiple edges and loops are not allowed, and we set $w_{i j}=0$ if $v_{i}$ and $v_{j}$ are not connected; otherwise, $w_{i j}>0$. Note that $w_{i i}=0$ and $w_{i j}=w_{j i}$. We will define the degree of a vertex $v_{j}$ to be

$$
\operatorname{deg}\left(v_{j}\right)=\sum_{i=0}^{n} w_{i j}
$$

and the parameter $g$ (the genus of the graph) to be

$$
g=1+\sum_{i<j} w_{i j}-|V|=\sum_{i<j} w_{i j}-n .
$$

Note that, if $R=\mathbb{Z}$, these definitions coincide with the usual definitions for the vertex degree and genus of a multigraph where $w_{i j}$ is the number of edges connecting vertices $v_{i}$ and $v_{j}$.

A divisor on $G$ is a function $D: V \rightarrow R$. The degree of a divisor $D$ is defined as

$$
\operatorname{deg}(D)=\sum_{v \in V} D(v)
$$

2010 AMS Mathematics subject classification. Primary 05C25, 14C40, 14 T05.

Keywords and phrases. Weighted graph, Riemann-Roch theorem.

Received by the editors on June 5, 2013, and in revised form on December 23, 2014. 
For any $x \in \mathbb{R}$, we say that $D>x$ if $D(v)>x$ for each $v \in V$, and $D>D^{\prime}$ if $D(v)>D^{\prime}(v)$ for each $v \in V$.

The space of divisors on $G$, written $\operatorname{Div}(G)$, is an $R$-module, and the subset of divisors on $G$ with degree 0 is denoted by $\operatorname{Div}^{0}(G)$.

The canonical divisor $K$ is defined by $K(v)=\operatorname{deg}(v)-2$ for any $v \in V$. Note that $\operatorname{deg}(K)=2 g-2$.

For any $j \in\{0, \ldots, n\}$, consider the divisor $H_{j}$, defined by

$$
H_{j}\left(v_{i}\right)= \begin{cases}\operatorname{deg}\left(v_{j}\right) & \text { if } v_{i}=v_{j}, \\ -w_{i j} & \text { otherwise. }\end{cases}
$$

Note that these $H_{j}$ 's are the columns of the edge-weighted Laplacian matrix of the graph. The principal divisors $\operatorname{PDiv}(G)$ are the $\mathbb{Z}$ linear combinations of the $H_{j}$ divisors. Two divisors $D, D^{\prime} \in \operatorname{Div}(G)$ are linearly equivalent, written $D \sim D^{\prime}$ if and only if there is an $H \in \operatorname{PDiv}(G)$ such that $D-D^{\prime}=H$.

For each divisor $D \in \operatorname{Div}(G)$, we associate a complete linear system $|D|$, which is defined as

$$
|D|=\left\{D^{\prime} \in \operatorname{Div}(G) \mid D^{\prime} \sim D, D^{\prime}>-1\right\},
$$

where the condition that $D^{\prime}>-1$ means that, at every vertex $v$, $D^{\prime}(v)>-1$. The reader may wonder why the condition of being strictly greater than -1 is the correct notion here, rather than the more attractive choice of being non-negative, which would be more akin to the notion of "effective" divisors. First, we note that there is no difference over the integers, which is the more commonly encountered area of application. More substantively, we prove that the RiemannRoch statement is simply false for the alternative definition. We present an example below. The dimension of $|D|$ is defined as:

$$
\ell(D)=\min _{E}\{\operatorname{deg}(E)|E \in \operatorname{Div}(G), E \geq 0,| D-E \mid=\emptyset\} .
$$

Now, we show the following Riemann-Roch formula holds on $G$.

Theorem 1.1. For any divisor $D \in \operatorname{Div}(G)$,

$$
\ell(D)-\ell(K-D)=\operatorname{deg}(D)+1-g .
$$


This theorem generalizes a similar statement for integral divisors on multigraphs proved by Baker and Norine [1]. In [5], we showed that Theorem 1.1 follows from the result in [1]. Here, the proof of Theorem 1.1 is independent from that of [5], and, although there are parallels with the Baker-Norine approach, we rely on the monotonicity of the Laplacian, which we believe provides an alternative route to the proof. Theorem 1.1 also resonates with the Riemann-Roch statements for tropical curves [4]; however, we do not see that either case implies the other. In tropical geometry, divisors are allowed to be associated with interior points of edges, and that is not our combinatorial construct.

Consider the two-vertex graph with edge weight of $3 / 2$; this graph has genus $g=1 / 2$. Using obvious ordered pair notation for divisors, we see that $K=(-1 / 2,-1 / 2)$. The group of principal divisors is generated by $(3 / 2,-3 / 2)$. Consider the divisor $D=(1 / 2,-1 / 2)$; then $K-D=(-1,0)$. If we insist on using the notion of a linear system based on nonnegative divisors (instead of the $>-1$ condition), then it is easy to see that both $|D|$ and $|K-D|$ are empty, and have $\ell(D)=\ell(K-D)=0$. Hence, Riemann-Roch would fail to be true:

$$
0-0 \neq 0+1-g .
$$

Define the set of all divisors of degree $g-1$ with empty linear systems by:

$$
\mathcal{N}(G)=\{D \in \operatorname{Div}(G) \mid \operatorname{deg}(D)=g-1 \text { and }|D|=\emptyset\} .
$$

\section{Theorem 1.2.}

(i) The set $\mathcal{N}(G)$ is symmetric with respect to $K$; that is, $N \in \mathcal{N}(G)$ if and only if $K-N \in \mathcal{N}(G)$.

(ii) For any $D \in \operatorname{Div}(G),|D|=\emptyset$ if and only if there is an $N \in \mathcal{N}(G)$ such that $D \leq N$.

The proof of Theorem 1.2, which relies on a normal form for divisors, up to linear equivalence, follows in the subsequent sections.

2. Proof of Riemann-Roch. For any $D \in \operatorname{Div}(G)$, define

$$
D^{+}(v)=\max (D(v), 0), \quad D^{-}(v)=\min (D(v), 0) .
$$


It follows directly from these definitions that, for any $D \in \operatorname{Div}(G)$,

$$
D=D^{+}+D^{-} \quad \text { and } \quad \operatorname{deg}\left(D^{+}\right)=-\operatorname{deg}\left((-D)^{-}\right) .
$$

Lemma 2.1. If Theorem 1.2 (ii) is true, then, for any $D \in \operatorname{Div}(G)$,

$$
\ell(D)=\min _{N \in \mathcal{N}(G)} \operatorname{deg}\left((D-N)^{+}\right)
$$

Proof. The definition of $\ell(D)$ is:

$$
\ell(D)=\min _{E}\{\operatorname{deg}(E)|E \geq 0,| D-E \mid=\emptyset .
$$

Theorem 1.2 (ii) implies that

$$
\begin{aligned}
\ell(D) & =\min _{E, N}\{\operatorname{deg}(E) \mid E \geq 0, N \in \mathcal{N}(G), D-E \leq N\} \\
& =\min _{E, N}\{\operatorname{deg}(E) \mid E \geq 0, N \in \mathcal{N}(G), E \geq D-N\},
\end{aligned}
$$

or equivalently,

$$
\ell(D)=\min _{N \in \mathcal{N}(G)} \operatorname{deg}\left((D-N)^{+}\right)
$$

Lemma 2.2. If Theorem 1.2 holds, then, for any $D \in \operatorname{Div}(G)$,

$$
\ell(K-D)=g-1-\operatorname{deg}(D)+\min _{M \in \mathcal{N}(G)} \operatorname{deg}\left((D-M)^{+}\right) .
$$

Proof. From Theorem 1.2 (ii),

$$
\begin{aligned}
\ell(K-D) & =\min _{E}\{\operatorname{deg}(E)|E \geq 0,| K-D-E \mid=\emptyset\} \\
& =\min _{E, M}\{\operatorname{deg}(E) \mid E \geq 0, M \in \mathcal{N}(G), K-D-E \leq M\} .
\end{aligned}
$$

If $K-D-E \leq M$ for $M \in \mathcal{N}(G)$, then $D+E \geq K-M$. Theorem 1.2 (i) implies that $K-M \in \mathcal{N}(G)$ if and only if $M \in \mathcal{N}(G)$; thus, we have:

$$
\begin{aligned}
\ell(K-D) & =\min _{E, M}\{\operatorname{deg}(E) \mid E \geq 0, M \in \mathcal{N}(G), D+E \geq M\} \\
& =\min _{E, M}\{\operatorname{deg}(E) \mid E \geq 0, M \in \mathcal{N}(G), E \geq M-D\} \\
& =\min _{M \in \mathcal{N}(G)} \operatorname{deg}\left((M-D)^{+}\right) .
\end{aligned}
$$


Since

$$
\operatorname{deg}\left((M-D)^{+}\right)=\operatorname{deg}(M-D)-\operatorname{deg}\left((M-D)^{-}\right)
$$

we have:

$$
\begin{aligned}
\ell(K-D) & =\min _{M \in \mathcal{N}(G)} \operatorname{deg}\left((M-D)^{+}\right) \\
& =\min _{M \in \mathcal{N}(G)}\left(\operatorname{deg}(M-D)-\operatorname{deg}\left((M-D)^{-}\right)\right) \\
& =\operatorname{deg}(M)-\operatorname{deg}(D)+\min _{M \in \mathcal{N}(G)}\left(-\operatorname{deg}\left((M-D)^{-}\right)\right) \\
& =g-1-\operatorname{deg}(D)+\min _{M \in \mathcal{N}(G)} \operatorname{deg}\left((D-M)^{+}\right) .
\end{aligned}
$$

We now have the ingredients to prove Theorem 1.1.

Proof of Theorem 1.1. Using Lemmas 2.1 and 2.2, we have

$$
\begin{aligned}
\ell(D)-\ell(K-D)= & \left(\min _{N \in \mathcal{N}(G)} \operatorname{deg}\left((D-N)^{+}\right)\right) \\
& -\left(g-1-\operatorname{deg}(D)+\min _{M \in \mathcal{N}(G)} \operatorname{deg}\left((D-M)^{+}\right)\right) \\
= & \operatorname{deg}(D)-g+1+\min _{N \in \mathcal{N}(G)} \operatorname{deg}\left((D-N)^{+}\right) \\
& -\min _{M \in \mathcal{N}(G)} \operatorname{deg}\left((D-M)^{+}\right)=\operatorname{deg}(D)-g+1 .
\end{aligned}
$$

3. Reduced divisors. Let $V_{0}=V-\left\{v_{0}\right\}$. We say that a divisor $D \in \operatorname{Div}(G)$ is reduced if and only if:

(i) $D(v)>-1$ for each $v \in V_{0}$, and,

(ii) for every $I \subset\{1, \ldots, n\}$, there is a $v \in V_{0}$ such that

$$
\left(D-\sum_{j \in I} H_{j}\right)(v) \leq-1 .
$$

Define $\mathcal{P}(G) \subset \operatorname{PDiv}(G)$ to be the set of non negative, non-zero $\mathbb{Z}$ linear combinations of the $H_{j}$ divisors for $j>0$; that is, if $H \in \mathcal{P}(G)$, then there is a set of nonnegative integers $\left\{c_{1}, \ldots, c_{n}\right\}$ with at least one $c_{j}>0$ such that

$$
H=\sum_{j=1}^{n} c_{j} H_{j} .
$$


Lemma 3.1. Suppose a divisor $D(v)>-1$ for all $v \in V_{0}$; then $D$ is reduced if and only if, for every $H \in \mathcal{P}(G)$, there is a $v \in V_{0}$ such that

$$
(D-H)(v) \leq-1 .
$$

Proof. Assume that $D(v)>-1$ for all $v \in V_{0}$. If, for every $H \in \mathcal{P}(G)$, there is a $v \in V_{0}$ such that $(D-H)(v) \leq-1$, then $D$ is clearly reduced; thus, we only need to show that the converse is true.

Suppose that there exists:

$$
H=\sum_{i=1}^{n} c_{i} H_{i} \in \mathcal{P}(G),
$$

such that $(D-H)(v)>-1$ for all $v \in V_{0}$. This means that, for each $j=1, \ldots, n$,

$$
D\left(v_{j}\right)>c_{j} \operatorname{deg}\left(v_{j}\right)-\sum_{i=1}^{n} c_{i} w_{i j}-1
$$

Let $\alpha=\max \left\{c_{1}, \ldots, c_{n}\right\}$ and, for each $i=1, \ldots, n$, set

$$
b_{i}= \begin{cases}1 & \text { if } c_{i}=\alpha \\ 0 & \text { otherwise }\end{cases}
$$

We claim that, for each $j \in\{1, \ldots, n\}$,

$$
D\left(v_{j}\right)>b_{j} \operatorname{deg}\left(v_{j}\right)-\sum_{i=1}^{n} b_{i} w_{i j}-1 .
$$

If $b_{j}=0$, we have:

$$
b_{j} \operatorname{deg}\left(v_{j}\right)-\sum_{i=1}^{n} b_{i} w_{i j}-1=-\sum_{i=1}^{n} b_{i} w_{i j}-1 \leq-1<D\left(v_{j}\right) .
$$

Define the index sets $A_{j}$ and $B_{j}$ as:

$$
\begin{aligned}
& A_{j}=\left\{i>0 \mid w_{i j}>0 \text { and } c_{i}<\alpha\right\}, \\
& B_{j}=\left\{i>0 \mid w_{i j}>0 \text { and } c_{i}=\alpha\right\} .
\end{aligned}
$$

Note that:

$$
\sum_{i=1}^{n} c_{i} w_{i j}=\sum_{i \in A_{j}} c_{i} w_{i j}+\alpha \sum_{i \in B_{j}} w_{i j}
$$


If $b_{j}=1$, then $c_{j}=\alpha$ and

$$
\begin{aligned}
D\left(v_{j}\right) & >c_{j} \operatorname{deg}\left(v_{j}\right)-\sum_{i=1}^{n} c_{i} w_{i j}-1 \\
& =\alpha \operatorname{deg}\left(v_{j}\right)-\sum_{i \in A_{j}} c_{i} w_{i j}-\alpha \sum_{i \in B_{j}} w_{i j}-1 \\
& =\alpha\left(w_{0 j}+\sum_{i \in A_{j}} w_{i j}+\sum_{i \in B_{j}} w_{i j}\right)-\sum_{i \in A_{j}} c_{i} w_{i j}-\alpha \sum_{i \in B_{j}} w_{i j}-1 \\
& =\alpha w_{0 j}+\sum_{i \in A_{j}}\left(\alpha-c_{i}\right) w_{i j}-1 \geq w_{0 j}+\sum_{i \in A_{j}} w_{i j}-1 .
\end{aligned}
$$

Also, we have:

$$
\begin{aligned}
b_{j} \operatorname{deg}\left(v_{j}\right)-\sum_{i=1}^{n} b_{i} w_{i j}= & \operatorname{deg}\left(v_{j}\right)-\sum_{i \in B_{j}} w_{i j}=w_{0 j}+\sum_{i \in A_{j}} w_{i j} \\
& +\sum_{i \in B_{j}} w_{i j}-\sum_{i \in B_{j}} w_{i j}=w_{0 j}+\sum_{i \in A_{j}} w_{i j} .
\end{aligned}
$$

Thus,

$$
D\left(v_{j}\right)>b_{j} \operatorname{deg}\left(v_{j}\right)-\sum_{i=1}^{n} b_{i} w_{i j}-1,
$$

for each $j=1, \ldots, n$; hence, $D$ is not reduced.

Let $\Delta_{0}$ be the edge-weighted reduced Laplacian of $G$, which can be represented by the $n \times n$ matrix:

$$
\Delta_{0}=\left(\begin{array}{cccc}
\operatorname{deg}\left(v_{1}\right) & -w_{12} & \cdots & -w_{1 n} \\
-w_{12} & \operatorname{deg}\left(v_{2}\right) & \cdots & -w_{2 n} \\
\vdots & \vdots & \ddots & \vdots \\
-w_{1 n} & -w_{2 n} & \cdots & \operatorname{deg}\left(v_{n}\right)
\end{array}\right) .
$$

For $x=\left(x_{1}, \ldots, x_{n}\right)$ and $y=\left(y_{1}, \ldots, y_{n}\right)$, we say that $x>y$ if and only if $x_{i}>y_{i}$ for each $i$. For any scalar $a \in \mathbb{R}, x>a$ if and only if $x_{i}>a$ for each $i$. We define:

$$
\max (x, y)=\left(\max \left\{x_{1}, y_{1}\right\}, \ldots, \max \left\{x_{n}, y_{n}\right\}\right)
$$


and

$$
\min (x, y)=\left(\min \left\{x_{1}, y_{1}\right\}, \ldots, \min \left\{x_{n}, y_{n}\right\}\right) .
$$

In [5], we showed that $\Delta_{0}$ is monotone, that is, for any $x \in \mathbb{R}^{n}$, if $\Delta_{0} x \geq 0$, then $x \geq 0$. Monotonicity implies that $\Delta_{0}^{-1}$ exists and is nonnegative, and that if $x, y \geq 0$ with $y \geq \Delta_{0} x$, then $\Delta_{0}^{-1} y \geq x$ (see [2]).

Lemma 3.2. For any $z \in \mathbb{R}^{n}$ such that $z \geq 0$, there is a $c \in \mathbb{Z}^{n}$ such that $c \geq 0$ and $\Delta_{0} c \geq z$.

Proof. Fix $z \in \mathbb{R}^{n}$ with $z \geq 0$. Let

$$
C_{0}=\left\{x \in \mathbb{R}^{n} \mid \Delta_{0} x \geq 0\right\}, \quad C_{z}=\left\{x \in \mathbb{R}^{n} \mid \Delta_{0} x \geq z\right\}
$$

and

$$
K=\left\{x \in \mathbb{R}^{n} \mid x \geq 0\right\} .
$$

Since $\Delta_{0}$ is monotone, $C_{z} \neq \emptyset$ and $C_{z} \subset C_{0} \subset K$.

Letting $x, y \in C_{0}$ and $\alpha, \beta \in \mathbb{R}$ with $\alpha, \beta \geq 0$, then

$$
\Delta_{0}(\alpha x+\beta y)=\alpha \Delta_{0} x+\beta \Delta_{0} y \geq 0,
$$

and $\alpha x+\beta y \in C_{0}$. Thus, $C_{0}$ is a convex cone, and, since $\Delta_{0}$ is injective, $C_{0}$ has an interior. Let $v=\Delta_{0}^{-1} z$. For any $x \in C_{z}$,

$$
\Delta_{0}(x-v)=\Delta_{0} x-z \geq 0,
$$

so $x-v \in C_{0}$ and $C_{z}-v$ is also a convex cone; thus, $C_{z}$ is a convex affine cone with an interior. Hence, $C_{z} \cap \mathbb{Z}^{n} \neq \emptyset$.

Define the function $\phi: \operatorname{Div}(G) \rightarrow R^{n}$ as:

$$
\phi(D)=\left(D\left(v_{1}\right), \ldots, D\left(v_{n}\right)\right) .
$$

We can represent any

$$
H=\sum_{i=1}^{n} c_{i} H_{i} \in \operatorname{PDiv}(G)
$$

as:

$$
\phi(H)=\Delta_{0} c,
$$


where $c=\left(c_{1}, \ldots, c_{n}\right) \in \mathbb{Z}^{n} . H\left(v_{0}\right)$ can be recovered by:

$$
H\left(v_{0}\right)=\sum_{i=1}^{n} c_{i} H_{i}\left(v_{0}\right)=-\sum_{i=1}^{n} c_{i} w_{0 i} .
$$

For any $d \in R^{n}$, define $\mathcal{A}(d) \subset \mathbb{Z}^{n}$ as:

$$
\mathcal{A}(d)=\left\{c \in \mathbb{Z}^{n} \mid c \geq 0 \quad \text { and } \quad \Delta_{0} c<d\right\} .
$$

Note that, if $d>0$, then $\mathcal{A}(d) \neq \emptyset$, since the zero vector $(0, \ldots, 0) \in$ $\mathcal{A}(d)$. Also, note that, for any $c \in \mathcal{A}(d)$ such that $c \neq 0$, there is a corresponding $H \in \mathcal{P}(G)$ such that $\phi(H)=\Delta_{0} c$.

Using the above notation, we can restate Lemma 3.1 in terms of $n$-tuples in $R^{n}$ as follows. Suppose that $D \in \operatorname{Div}(G)$ with $D(v)>-1$ for all $v \in V_{0}$. Set $d=\phi(D+1)$; thus, $d>0$ and, if $D$ is reduced, the condition $(D-H)(v) \leq-1$ for some $v \in V_{0}$ for each $H \in \mathcal{P}(G)$ is equivalent to $d \ngtr \Delta_{0} c$ for all nonzero $c \in \mathcal{A}(d)$. Thus, $\mathcal{A}(d)=\{0\}$, the set containing only the zero vector in $\mathbb{Z}^{n}$.

Lemma 3.3. Let $D \in \operatorname{Div}(G)$, and set $d=\phi(D+1)$. If $d>0$, then $\mathcal{A}(d)$ is bounded; that is, there exists a $b \in \mathbb{R}^{n}$ such that $b \geq c$ for all $c \in \mathcal{A}(d)$.

Proof. Suppose $d>0$ and $c \in \mathcal{A}(d)$. Since $d>\Delta_{0} c$ and $\Delta_{0}$ is monotone, we have $b=\Delta_{0}^{-1} d \geq c$.

Lemma 3.4. Let $D \in \operatorname{Div}(G)$, and set $d=\phi(D+1)$. If $d>0$ and $c$, $c^{\prime} \in \mathcal{A}(d)$, then $\max \left(c, c^{\prime}\right) \in \mathcal{A}(d)$.

Proof. Suppose $d>0$ and $c, c^{\prime} \in \mathcal{A}(d)$. Then we have both $d-\Delta_{0} c>0$ and $d-\Delta_{0} c^{\prime}>0$. We can write the $j$ th component of $\Delta_{0} c$ as:

$$
\begin{aligned}
\left(\Delta_{0} c\right)_{j} & =c_{j} \operatorname{deg}\left(v_{j}\right)-\sum_{i=1}^{n} c_{i} w_{i j}=\sum_{i=0}^{n} c_{j} w_{i j}-\sum_{i=1}^{n} c_{i} w_{i j} \\
& =c_{j} w_{0 j}+\sum_{i=1}^{n}\left(c_{j}-c_{i}\right) w_{i j}
\end{aligned}
$$


and similarly for $\Delta_{0} c^{\prime}$. For $d=\left(d_{1}, \ldots, d_{n}\right)$, we have:

$$
d_{j}>c_{j} w_{0 j}+\sum_{i=1}^{n}\left(c_{j}-c_{i}\right) w_{i j}, \quad d_{j}>c_{j}^{\prime} w_{0 j}+\sum_{i=1}^{n}\left(c_{j}^{\prime}-c_{i}^{\prime}\right) w_{i j},
$$

for each $j$. If $\max \left\{c_{j}, c_{j}^{\prime}\right\}=c_{j}$, then

$$
d_{j}>c_{j} w_{0 j}+\sum_{i=1}^{n}\left(c_{j}-\max \left\{c_{i}, c_{i}^{\prime}\right\}\right) w_{i j},
$$

and, if $\max \left\{c_{j}, c_{j}^{\prime}\right\}=c_{j}^{\prime}$,

$$
d_{j}>c_{j}^{\prime} w_{0 j}+\sum_{i=1}^{n}\left(c_{j}^{\prime}-\max \left\{c_{i}, c_{i}^{\prime}\right\}\right) w_{i j} .
$$

We can combine these two relations to obtain

$$
d_{j}>\max \left\{c_{j}, c_{j}^{\prime}\right\} w_{0 j}+\sum_{i=1}^{n}\left(\max \left\{c_{j}, c_{j}^{\prime}\right\}-\max \left\{c_{i}, c_{i}^{\prime}\right\}\right) w_{i j},
$$

for each $j>0$. Thus,

$$
d-\Delta_{0} \max \left(c, c^{\prime}\right)>0,
$$

and $\max \left(c, c^{\prime}\right) \in \mathcal{A}(d)$.

Theorem 3.5. For any $D \in \operatorname{Div}(G)$, there is a unique $D_{0} \sim D$ such that $D_{0}$ is reduced.

Proof. Let $D \in \operatorname{Div}(G)$. By Lemma 3.2, choose $c \in \mathbb{Z}^{n}$ so that $\Delta_{0} c \geq \phi\left(-\left(D^{-}\right)\right)$, noting that $-\left(D^{-}\right) \geq 0$. Set $d=\phi(D+1)+\Delta_{0} c$, which guarantees that $d>0$.

By Lemmas 3.3 and 3.4, $\widehat{c}=\max \left\{c^{\prime} \mid c^{\prime} \in \mathcal{A}(d)\right\}$ exists and is unique. We claim that $\mathcal{A}\left(d-\Delta_{0} \widehat{c}\right)=\{0\}$. Letting $c^{\prime} \in \mathcal{A}\left(d-\Delta_{0} \widehat{c}\right)$, then

$$
d-\Delta_{0} \widehat{c}-\Delta_{0} c^{\prime}=d-\Delta_{0}\left(\widehat{c}+c^{\prime}\right)>0 ;
$$

thus, $\widehat{c}+c^{\prime} \in \mathcal{A}(d)$. Since $\widehat{c}$ is maximal in $\mathcal{A}(d)$ and $\widehat{c}, c^{\prime} \geq 0$, we must have $c^{\prime}=0$. From Lemma 3.1, it follows that there is a unique reduced divisor $D_{0}$ such that $\phi\left(D_{0}\right)=d-\Delta_{0} \widehat{c}$, where $D_{0} \sim D$ since the translations $\Delta_{0} c$ and $\Delta_{0} \widehat{c}$ correspond to some $H \in \operatorname{PDiv}(G)$. 
4. Empty linear systems. In this section, we will exploit properties of reduced divisors to determine the set of divisors which have empty linear systems. We begin with the following property of reduced divisors.

Lemma 4.1. If $D \in \operatorname{Div}(G)$ is reduced, then

$$
\sum_{v \in V_{0}} D(v) \leq g
$$

with equality if and only if there exists a permutation $\left(j_{1}, j_{2}, \ldots, j_{n}\right)$ of $(1,2, \ldots, n)$ such that

$$
D\left(v_{j_{k}}\right)=\sum_{i=0}^{k-1} w_{j_{i} j_{k}}-1
$$

for each $k=1, \ldots, n$, where $j_{0}=0$.

Proof. Suppose that $D$ is reduced. Then, for every $I \subset\{1, \ldots, n\}$, there is a $j \in V_{0}$ such that

$$
D\left(v_{j}\right) \leq \operatorname{deg}\left(v_{j}\right)-\sum_{i \in I} w_{i j}-1=\sum_{i \notin I} w_{i j}-1 .
$$

Suppose that $I=I_{0}=\{1, \ldots, n\}$, and that equation (4.1) is satisfied for $j=j_{1} \in I_{0}$. Then,

$$
D\left(v_{j_{1}}\right) \leq \sum_{i \notin I_{0}} w_{i j_{1}}-1=w_{0 j_{1}}-1
$$

Now, letting $I=I_{1}=I_{0}-\left\{j_{1}\right\}$, then equation (4.1) is satisfied for $j=j_{2} \in I_{1}$ so that

$$
D\left(v_{j_{2}}\right) \leq \sum_{i \notin I_{1}} w_{i j_{2}}-1=w_{0 j_{2}}+w_{j_{1} j_{2}}-1 .
$$

Similarly, for $I=I_{2}=I_{1}-\left\{j_{2}\right\}$, equation (4.1) is satisfied for $j=j_{3} \in I_{2}$ and

$$
D\left(v_{j_{3}}\right) \leq \sum_{i \notin I_{2}} w_{i j_{3}}-1=w_{0 j_{3}}+w_{j_{1} j_{3}}+w_{j_{2} j_{3}}-1 .
$$


Continuing this process, let $I_{k}=I_{k-1}-\left\{j_{k}\right\}$ for $k=1, \ldots, n-1$, where $j=j_{k} \in I_{k-1}$ satisfies equation (4.1) for $I_{k-1}$, and, in general, we have:

$$
D\left(v_{j_{k}}\right) \leq \sum_{i=0}^{k-1} w_{j_{i} j_{k}}-1
$$

where $j_{0}=0$. Note that the resulting $n$-tuple $\left(j_{1}, j_{2}, \ldots, j_{n}\right)$ is a permutation of $(1,2, \ldots, n)$. If we rewrite equation (4.2) as

$$
D\left(v_{j_{k}}\right)-\sum_{i=0}^{k-1} w_{j_{i} j_{k}}+1 \leq 0,
$$

and sum over all $k$, we have

$$
\sum_{k=1}^{n}\left(D\left(v_{j_{k}}\right)-\sum_{i=0}^{k-1} w_{j_{i} j_{k}}+1\right)=\sum_{j=1}^{n} D\left(v_{j}\right)-\sum_{i<j} w_{i j}+n \leq 0,
$$

or equivalently,

$$
\sum_{v \in V_{0}} D(v) \leq \sum_{i<j} w_{i j}-n=g .
$$

For the equality condition, we assume again that the $D$ is reduced and note that, if

$$
D\left(v_{j_{k}}\right)=\sum_{i=0}^{k-1} w_{j_{i} j_{k}}-1
$$

holds for some $\left(j_{1}, \ldots, j_{n}\right)$ for each $k=1, \ldots, n$, then

$$
\sum_{v \in V_{0}} D(v)=g
$$

follows directly since

$$
\sum_{i<j} w_{i j}-n=g .
$$

For the other direction, if

$$
\sum_{v \in V_{0}} D(v)=g,
$$


since $D$ is reduced, equation (4.2) holds at each $k$ for some permutation $\left(j_{1}, \ldots, j_{n}\right)$; thus, the only way that we can have equality is

$$
D\left(v_{j_{k}}\right)=\sum_{i=0}^{k-1} w_{j_{i} j_{k}}-1,
$$

for each $k=1, \ldots, n$.

An immediate application of Theorem 3.5 and Lemma 4.1 provides a sufficient condition for a divisor to have a nonempty linear system.

Lemma 4.2. Let $D \in \operatorname{Div}(G)$. If $\operatorname{deg}(D)>g-1$, then $|D| \neq \emptyset$.

Proof. Let $D$ be a divisor with $\operatorname{deg}(D)>g-1$, and let $D_{0}$ be the unique reduced divisor such that $D_{0} \sim D$, from Theorem 3.5. By Lemma 4.1,

$$
\sum_{v \in V_{0}} D_{0}(v) \leq g
$$

By assumption, we have:

$$
\operatorname{deg}(D)=\operatorname{deg}\left(D_{0}\right)=D_{0}\left(v_{0}\right)+\sum_{v \in V_{0}} D_{0}(v)>g-1
$$

or equivalently,

$$
D_{0}\left(v_{0}\right)>-\sum_{v \in V_{0}} D_{0}(v)+g-1 ;
$$

thus, $D_{0}\left(v_{0}\right)>-1$. Since $D_{0}(v)>-1$ for each $v \in V_{0},|D| \neq \emptyset$.

Lemma 4.3. If $D_{0}$ is a reduced divisor, then $\left|D_{0}\right| \neq \emptyset$ if and only if $D_{0}\left(v_{0}\right)>-1$.

Proof. Let $D_{0} \in \operatorname{Div}(G)$ be reduced. If $D_{0}\left(v_{0}\right)>-1$, then $D_{0}(v)>$ -1 for all $v \in V$ and $\left|D_{0}\right| \neq \emptyset$.

Now, assume that $\left|D_{0}\right| \neq \emptyset$; thus, there is a $P \in \operatorname{PDiv}(G)$ such that $D_{0}+P>-1$. Since $D_{0}$ is reduced, the only $P \in \operatorname{PDiv}(G)$ which would satisfy $D_{0}+P>-1$ must have $P(v) \geq 0$ for all $v \in V_{0}$. Since $\operatorname{deg}(P)=0$ and $P\left(v_{0}\right) \leq 0$, we must have $D_{0}>-1$ in order for $\left|D_{0}\right|$ to be nonempty. 
Lemma 4.4. If $D_{0}$ is a reduced divisor with $\operatorname{deg}\left(D_{0}\right)=g-1$ and $\left|D_{0}\right|=\emptyset$, then

$$
D_{0}\left(v_{j_{l}}\right)= \begin{cases}-1 & l=0 \\ \sum_{i=0}^{l-1} w_{j_{i} j_{l}}-1 & l>0\end{cases}
$$

where $j_{0}=0$ and $\left(j_{1}, \ldots, j_{n}\right)$ is a permutation of $(1, \ldots, n)$.

Proof. By Lemma 4.3, $D_{0}\left(v_{0}\right) \leq-1$; thus, by Lemma 4.1 we have:

$$
\sum_{i=1}^{n} D_{0}\left(v_{i}\right)=g,
$$

$D_{0}\left(v_{0}\right)=-1$ and

$$
D_{0}\left(v_{j_{l}}\right)=\sum_{i=0}^{l-1} w_{j_{i} j_{l}}-1
$$

for some permutation $\left(j_{1}, \ldots, j_{n}\right)$ of $(1, \ldots, n)$.

We will denote the reduced divisors in Lemma 4.4 as:

$\mathcal{N}_{0}(G)=\{D \in \operatorname{Div}(G)|| D \mid=\emptyset, \operatorname{deg}(D)=g-1, D$ is reduced $\} \subset \mathcal{N}(G)$, noting that $\left|\mathcal{N}_{0}(G)\right| \leq n$ !

A direct consequence of Lemma 4.4 gives us the composition of $\mathcal{N}(G)$, which is a lattice generated by $\mathcal{N}_{0}(G)$.

Lemma 4.5. $\mathcal{N}(G)=\left\{D \in \operatorname{Div}(G) \mid D \sim D_{0}\right.$ where $\left.D_{0} \in \mathcal{N}_{0}(G)\right\}$.

Proof. If $D \in \mathcal{N}(G)$, then by Lemma 3.5 there is a $D_{0} \in \mathcal{N}_{0}(G)$ which is linearly equivalent to $D$.

Now, we prove Theorem 1.2.

Proof of Theorem 1.2.

(i) Since any $D \in \mathcal{N}(G)$ can be written as $D=N_{0}+P$ for some $P \in \operatorname{PDiv}(G)$ and $N_{0} \in \mathcal{N}_{0}(G)$, it is sufficient to assume $D=N_{0}$. 
By Lemma 4.4,

$$
N_{0}\left(v_{j_{0}}\right)=-1 \quad \text { and } \quad N_{0}\left(v_{j_{k}}\right)=\sum_{i=0}^{k-1} w_{j_{i} j_{k}}-1
$$

for some permutation $\left(j_{1}, \ldots, j_{n}\right)$ of $(1, \ldots, n)$ with $j_{0}=0$. Since

$$
K\left(v_{i}\right)=\sum_{j=0}^{n} w_{i j}-2
$$

for $k>0$, we have

$$
(K-D)\left(v_{j_{k}}\right)=\sum_{i=0}^{n} w_{j_{i} j_{k}}-2-\sum_{i=0}^{k-1} w_{j_{i} j_{k}}+1=\sum_{i=k}^{n} w_{j_{i} j_{k}}-1,
$$

and, for $k=0$,

$$
(K-D)\left(v_{j_{0}}\right)=\sum_{i=0}^{n} w_{j_{i} j_{0}}-1=\operatorname{deg}\left(v_{j_{0}}\right)-1 .
$$

If we subtract $H_{0} \in \operatorname{PDiv}(G)$ from $K-D$, we have

$$
\left(K-D-H_{0}\right)\left(v_{j_{0}}\right)=-1
$$

and, for $k>0$,

$$
\left(K-D-H_{0}\right)\left(v_{j_{k}}\right)=\sum_{i=k}^{n} w_{j_{i} j_{k}}-1+w_{j_{0} j_{k}} .
$$

Let $l_{0}=j_{0}=0$ and $l_{k}=j_{n-k+1}$ for $k=1, \ldots, n$; then, $\left(l_{1}, \ldots, l_{n}\right)$ is a permutation of $(1, \ldots, n)$, and

$$
\left(K-D-H_{0}\right)\left(v_{l_{k}}\right)=\sum_{i=0}^{k-1} w_{l_{i} l_{k}}-1 .
$$

Thus, $K-D-H_{0} \in \mathcal{N}_{0}(G)$ and $K-D \in \mathcal{N}(G)$.

Now, assume that $K-D \in \mathcal{N}_{0}(G)$. Let $D^{\prime}=K-D$, and from the above, we have $K-D^{\prime}=D \in \mathcal{N}(G)$.

(ii) Let $D \in \operatorname{Div}(G)$ with $|D|=\emptyset$. By Lemma 3.5, there is a unique reduced divisor $D_{0} \sim D$. Since $\left|D_{0}\right|=\emptyset$, Lemma 4.3 implies that $D_{0}\left(v_{0}\right) \leq-1$. By the proof of Lemma 4.1, we have that equation (4.2) 
holds for each $D_{0}(v)$ where $v \in V_{0}$, so for some permutation $\left(j_{1}, \ldots, j_{n}\right)$ of $(1, \ldots, n)$,

$$
D_{0}\left(v_{j_{k}}\right) \leq \sum_{i=0}^{k-1} w_{j_{i} j_{k}}-1 .
$$

Thus, $D_{0} \leq N_{0}$ for one of the $N_{0} \in \mathcal{N}_{0}(G)$. Let $P \in \operatorname{PDiv}(G)$ be such that $D=D_{0}+P$, and let $N=N_{0}+P$. Then, we have $D \leq N$ where $N \in \mathcal{N}(G)$.

\section{REFERENCES}

1. M. Baker and S. Norine, Riemann-Roch and Abel-Jacobi theory on a finite graph, Adv. Math. 215 (2007), 766-788.

2. A. Berman and R.J. Plemmons, Nonnegative matrices in the mathematical sciences, SIAM, Philadelphia, PA, 1994.

3. N.L. Biggs, Chip-firing and the critical group of a graph, J. Alg. Comb. 9 (1999), 25-45.

4. A. Gathmann and M. Kerber, A Riemann-Roch theorem in tropical geometry, Math. Z. 259 (2008), 217-230.

5. R. James and R. Miranda, A Riemann-Roch theorem for edge-weighted graphs, Proc. Amer. Math. Soc. 141 (2013), 3793-3802.

6. A. Postnikov and B. Shapiro, Trees, parking functions, syzygies, and deformations of monomial ideals, Trans. Amer. Math. Soc. 356 (2004), 3109-3142.

Department of Mathematics and Computer Science, Colorado College, Colorado Springs, CO 80523

Email address: rodney.james@coloradocollege.edu

Department of Mathematics, Colorado State University, Fort Collins, CO 80523

Email address: rick.miranda@colostate.edu 